\title{
A Criterion for linear independence of infinite products
}

\author{
Jaroslav Hančl, ${ }^{*}$ Ondřej Kolouch ${ }^{\dagger}$ and Lukáš Novotný ${ }^{\dagger}$
}

\begin{abstract}
Using an idea of Erdős the paper establishes a criterion for the linear independence of infinite products which consist of rational numbers. A criterion for irrationality is obtained as a consequence.
\end{abstract}

\section{Introduction}

Following Erdős [1] we prove

Theorem 1.1. Let $K$ be a non-negative integer and let $\left\{a_{n}\right\}_{n=1}^{\infty}$ be a nondecreasing sequence of positive integers such that

$$
1<\liminf _{n \rightarrow \infty} a_{n}^{\frac{1}{(K+2)^{n}}}<\limsup _{n \rightarrow \infty} a_{n}^{\frac{1}{(K+2)^{n}}}<\infty .
$$

Then the numbers $1, \prod_{n=1}^{\infty}\left(1+\frac{1}{a_{n}+1}\right), \prod_{n=1}^{\infty}\left(1+\frac{1}{n a_{n}+1}\right), \cdots$, and $\prod_{n=1}^{\infty}(1+$ $\left.\frac{1}{n^{K} a_{n}+1}\right)$ are linearly independent over the rational numbers.

As a consequence of this theorem we obtain a criterion for infinite products to be irrational.

Key Words: Linear independence, infinite product, irrationality.

2010 Mathematics Subject Classification: 11J72.

Received: October, 2013.

Revised: December, 2013.

Accepted: December, 2013.

*This work was supported by the European Regional Development Fund in the IT4Innovations Centre of Excellence project (CZ.1.05/1.1.00/02.0070) and by grants no. P201/12/2351 and MSM 6198898701.

${ }^{\dagger}$ The authors were supported by grants $01798 / 2011 / R R C$ and $02508 / 2013 / R R C$ of the Moravian-Silesian region and by SGS08/PřF/2014. 
Theorem 1.2. Let $\left\{a_{n}\right\}_{n=1}^{\infty}$ be a non-decreasing sequence of positive integers such that

$$
1<\liminf _{n \rightarrow \infty} a_{n}^{\frac{1}{2^{n}}}<\limsup _{n \rightarrow \infty} a_{n}^{\frac{1}{2^{n}}}<\infty .
$$

Then the number $\prod_{n=1}^{\infty}\left(1+\frac{1}{a_{n}}\right)$ is irrational.

The authors do not know if the number $\prod_{n=1}^{\infty}\left(1+\frac{1}{2^{2^{n}} a_{n}+1}\right)$ is irrational for all sequences $\left\{a_{n}\right\}_{n=1}^{\infty}$ of positive integers although we know from another theorem of Erdős [1] that the number $\sum_{n=1}^{\infty} \frac{1}{2^{2^{n}} a_{n}}$ is irrational for every sequence $\left\{a_{n}\right\}_{n=1}^{\infty}$ of positive integers. Hančl and Kolouch [4] proved that if $\lim _{n \rightarrow \infty} a_{n}^{\frac{1}{2^{n}}}=\infty$ and $a_{n} \in \mathbb{Z}^{+}$then the number $\prod_{n=1}^{\infty}\left(1+\frac{1}{a_{n}}\right)$ is irrational, but we do not know if it is transcendental.

It is not difficult to prove that $\prod_{n=1}^{\infty}\left(1+\frac{1}{2^{2^{n}}}\right)=\frac{4}{3}$, but we do not know if the number $\prod_{n=1}^{\infty}\left(1+\frac{1}{\left(2^{2^{n}}+1\right) a_{n}}\right)$ is irrational for all sequences $\left\{a_{n}\right\}_{n=1}^{\infty}$ of positive integers. Erdős [2] asked if the number $\sum_{n=1}^{\infty} \frac{1}{\left(2^{2^{n}}+1\right) a_{n}}$ is irrational for all sequences $\left\{a_{n}\right\}_{n=1}^{\infty}$ of positive integers.

A simple calculation shows that $\prod_{n=2}^{\infty}\left(1-\frac{1}{n^{2}}\right)=\frac{1}{2}$. On the other side the authors are not able to decide if the number $\prod_{n=1}^{\infty}\left(1+\frac{1}{n_{1}^{2}}\right)$ is irrational. In fact we are not able to prove that the number $\prod_{n=1}^{\infty}\left(1+\frac{1}{n^{k}}\right)$ is irrational for any $k \in \mathbb{Z}^{+}, k \neq 1$. This is analogous to the problem of the irrationality of the function $\zeta(k)=\sum_{n=1}^{\infty} \frac{1}{n^{k}}=\prod_{n=1}^{\infty}\left(1+\frac{1}{p_{n}^{k}-1}\right)$ for $k \in \mathbb{Z}^{+}, k \neq 1$ where $\left\{p_{n}\right\}_{n=1}^{\infty}$ is the increasing sequence of all primes. We know that for even $k$ the number $\zeta(k)$ is transcendental and that $\zeta(3)$ is an irrational number. But we do not know if the number $\prod_{n=1}^{\infty}\left(1+\frac{1}{p_{n}^{k}}\right)$ is irrational for any $k \in \mathbb{Z}^{+}, k \neq 1$.

We also do not know if the numbers $\prod_{n=1}^{\infty}\left(1+\frac{1}{n !}\right)$ and $\prod_{n=1}^{\infty}\left(1+\frac{n^{\pi(n)}}{n !}\right)$ are $\mathbb{Q}$-linearly independent where $\pi(n)$ denotes the number of primes less than or equal to $n$. Moreover, we do not know if the number $\prod_{n=1}^{\infty}\left(1+\frac{1}{n !}\right)$ is irrational.

There exists a nice book by Nishioka [6] which contains a review of results concerning the linear and algebraic independence of infinite products and series which use the strong tools of Mahler's method. Several general results concerning the linear independence of infinite series can be found in [3]. For other results in this theory see [5], for instance.

Our main theorem is Theorem 2.1 concerning $\mathbb{Q}$-linear independence. Its proof falls into two parts, the second part separated into two main cases, is based on the location of specific gap and makes use of some suitable tricks. As a consequence of Theorem 2.1 we obtain a criterion for irrationality in Theorem 2.2. Šustek [7] used similar method of Erdős to prove the irrationality measures.

We denote by $\mathbb{Z}, \mathbb{Z}^{+}, \mathbb{N}$, and $\mathbb{Q}$ the set of all integers, positive integers, nonnegative integers, and rational numbers, respectively. The functions $[x]$, 
and $\log _{2} x$ are the greatest integer less than or equal to $x$, and the logarithm to the base 2 of the number $x$, respectively. Notation $\log _{2}^{a} \log _{2} x$ means $\left(\log _{2}\left(\log _{2} x\right)\right)^{a}$.

\section{Main results}

Our first theorem is a basic result which deals with the $\mathbb{Q}$-linear independence of infinite products of rational numbers.

Theorem 2.1. Let $K$ be a positive integer and let $\varepsilon$ be a positive real number. Assume that $\left\{a_{i, n}\right\}_{n=1}^{\infty}$ and $\left\{b_{i, n}\right\}_{n=1}^{\infty}(i=1, \ldots, K)$ are sequences of positive integers such that $\left\{a_{1, n}\right\}_{n=1}^{\infty}$ is non-decreasing,

$$
\liminf _{n \rightarrow \infty} a_{1, n}^{\frac{1}{(K+1)^{n}}}<\limsup _{n \rightarrow \infty} a_{1, n}^{\frac{1}{(K+1)^{n}}}<\infty
$$

and

$$
\lim _{n \rightarrow \infty} \frac{a_{i, n} b_{j, n}}{b_{i, n} a_{j, n}}=0, \quad \text { for all } \quad j, i \in\{1, \ldots, K\}, \quad i>j .
$$

Suppose that for every sufficiently large number $n$

$$
\begin{gathered}
b_{i, n}<a_{1, n}^{\frac{1}{\log _{2}^{1+\varepsilon} \log _{2} a_{1, n}}}, \quad i=1, \ldots, K, \\
a_{1, n} \geq n^{1+\varepsilon},
\end{gathered}
$$

and

$$
a_{i, n} a_{1, n}^{-\frac{1}{\log _{2}^{1+\varepsilon} \log _{2} a_{1, n}}}<a_{1, n}<a_{i, n} a_{1, n}^{\frac{1}{\log _{2}^{1+\varepsilon} \log _{2} a_{1, n}}}, \quad i=2, \ldots, K .
$$

Then the products $\prod_{n=1}^{\infty}\left(1+\frac{b_{1, n}}{a_{1, n}}\right), \ldots$, and $\prod_{n=1}^{\infty}\left(1+\frac{b_{K, n}}{a_{K, n}}\right)$, and the number 1 are $\mathbb{Q}$-linearly independent.

Corollary 2.1. Let $K$ be a positive integer. Assume that $\left\{a_{i, n}\right\}_{n=1}^{\infty}$ and $\left\{b_{i, n}\right\}_{n=1}^{\infty}(i=1, \ldots, K)$ are sequences of positive integers such that $\left\{a_{1, n}\right\}_{n=1}^{\infty}$ is non-decreasing, $\left\{b_{i, n}\right\}_{n=1}^{\infty}(i=1, \ldots, K)$ is bounded,

$$
\liminf _{n \rightarrow \infty} \frac{1}{n} \log _{2} a_{1, n} \geq 1
$$

and

$$
\liminf _{n \rightarrow \infty} a_{1, n}^{\frac{1}{(K+1)^{n}}}<\limsup _{n \rightarrow \infty} a_{1, n}^{\frac{1}{(K+1)^{n}}}<\infty
$$


Suppose that for every $i \in\{2, \ldots, K\}$

$$
\lim _{n \rightarrow \infty} \frac{a_{i, n}}{a_{i-1, n}}=0
$$

and

$$
-1<\liminf _{n \rightarrow \infty} \frac{\log _{2}^{2} n}{n} \log _{2}\left(\frac{a_{1, n}}{a_{i, n}}\right) \leq \limsup _{n \rightarrow \infty} \frac{\log _{2}^{2} n}{n} \log _{2}\left(\frac{a_{1, n}}{a_{i, n}}\right)<1 .
$$

Then the products $\prod_{n=1}^{\infty}\left(1+\frac{b_{1, n}}{a_{1, n}}\right), \ldots$, and $\prod_{n=1}^{\infty}\left(1+\frac{b_{K, n}}{a_{K, n}}\right)$, and the number 1 are $\mathbb{Q}$-linearly independent.

Our second theorem is a consequence of the previous theorem and deals with the irrationality of infinite products over the rational numbers

Theorem 2.2. Let $\varepsilon$ be a positive real number. Suppose that $\left\{a_{n}\right\}_{n=1}^{\infty}$ and $\left\{b_{n}\right\}_{n=1}^{\infty}$ are two sequences of positive integers with $\left\{a_{n}\right\}_{n=1}^{\infty}$ non-decreasing and such that $\liminf _{n \rightarrow \infty} a_{n}^{\frac{1}{2^{n}}}<\limsup _{n \rightarrow \infty} a_{n}^{\frac{1}{2^{n}}}<\infty$. Assume that $a_{n} \geq$ $n^{1+\varepsilon}$ and $b_{n} \leq a_{n}^{\log _{2}^{-(1+\varepsilon)} \log _{2} a_{n}}$ hold for every large $n$. Then the product $\prod_{n=1}^{\infty}\left(1+\frac{b_{n}}{a_{n}}\right)$ is an irrational number.

Corollary 2.2. Assume that $\left\{a_{n}\right\}_{n=1}^{\infty}$ and $\left\{b_{n}\right\}_{n=1}^{\infty}$ are two sequences of positive integers such that $\left\{a_{n}\right\}_{n=1}^{\infty}$ is non-decreasing, $\liminf _{n \rightarrow \infty} \frac{1}{n} \log _{2} a_{n}>1$, $\left\{b_{n}\right\}_{n=1}^{\infty}$ is bounded and $\liminf _{n \rightarrow \infty} a_{n}^{\frac{1}{2^{n}}}<\lim \sup _{n \rightarrow \infty} a_{n}^{\frac{1}{2^{n}}}<\infty$. Then the product $\prod_{n=1}^{\infty}\left(1+\frac{b_{n}}{a_{n}}\right)$ is irrational.

Remark 2.1. From the simple calculation we obtain that $\prod_{n=1}^{\infty}\left(1+\frac{1}{2^{2^{n}}}\right)=\frac{4}{3}$. Hence the strict inequality between $\lim$ inf and $\lim \sup$ in all Theorems 1.1, 1.2, 2.1, 2.2 and Corollaries 2.1, 2.2 cannot be omited. It also shows implicitely that straightforward application of Liouville principle does not work.

\section{Proofs}

Theorem 1.1 is an immediate consequence of Theorem 2.1 when we set $K:=$ $K+1, a_{j, n}:=n^{K+1-j} a_{n}+1, b_{j, n}:=1$ for all $j \in\{1,2,3, \ldots, K+1\}$ and $n \in \mathbb{Z}^{+}$. The number $\varepsilon$ can be arbitrary because the sequence $\left\{a_{1, n}\right\}_{n=1}^{\infty}$ converges to infinity as a composition of two exponential functions.

Theorem 1.2 is an immediate consequence of Theorem 1.1 when we set $K:=0$ and $a_{n}:=a_{n}-1$ for all $n \in \mathbb{Z}^{+}$.

Theorem 2.2 is an immediate consequence of Theorem 2.1 when we set $K:=1$. Corollary 2.1 is an immediate consequence of Theorem 2.1 when we set $\varepsilon:=\frac{1}{2}$. 
Conditions (1)-(4) are fulfilled immediately. We only verify condition (5). From $\lim \inf _{n \rightarrow \infty} \frac{1}{n} \log _{2} a_{1, n} \geq 1$ we obtain that for all sufficiently large $n$ we have $a_{1, n}>2^{\frac{n}{2}}$. This and the fact that the function $x^{\frac{1}{\log _{2}^{1+\frac{1}{2}} \log _{2} x}}$ is increasing for a large $x$ imply that

$$
\frac{1}{a_{1, n}^{\log _{2}+\frac{1}{2}} \log _{2} a_{1, n}}>2^{\frac{n}{2} \frac{1}{\log _{2}^{1+\frac{1}{2}}\left(\frac{n}{2}\right)}}>2^{\frac{2 n}{\log _{2}^{2} n}} .
$$

Now for all $i \in\{2, \ldots, K\}$ and large $n$ we have

$$
-1<\liminf _{n \rightarrow \infty} \frac{\log _{2}^{2} n}{n} \log _{2}\left(\frac{a_{1, n}}{a_{i, n}}\right) \leq \limsup _{n \rightarrow \infty} \frac{\log _{2}^{2} n}{n} \log _{2}\left(\frac{a_{1, n}}{a_{i, n}}\right)<1 .
$$

Therefore

$$
2^{-\frac{2 n}{\log _{2}^{2} n}} a_{i, n}<a_{1, n}<a_{i, n} 2^{\frac{2 n}{\log _{2}^{2} n}} .
$$

From this and (6) we obtain (5).

Corollary 2.2 is an immediate consequence of Theorem 2.2 when we suppose in addition that $\left\{b_{n}\right\}_{n=1}^{\infty}$ is bounded,

Proof. (of Theorem 2.1) Set

$$
E=\liminf _{n \rightarrow \infty} a_{1, n}^{\frac{1}{(K+1)^{n}}} \text { and } \quad F=\limsup _{n \rightarrow \infty} a_{1, n}^{\frac{1}{(K+1)^{n}}}
$$

Assume that there is a $K$-tuple of integers $A_{1}, A_{2}, \ldots, A_{K}$ (not all equal to zero), $p \in \mathbb{Z}$ and $q \in \mathbb{Z}^{+}$such that

$$
\frac{p}{q}=\sum_{j=1}^{K} A_{j} \prod_{n=1}^{\infty}\left(1+\frac{b_{j, n}}{a_{j, n}}\right)=\sum_{j=1}^{R} A_{j} \prod_{n=1}^{\infty}\left(1+\frac{b_{j, n}}{a_{j, n}}\right)
$$

where $R$ is the largest index such that $A_{R} \neq 0$. Let $N \in \mathbb{Z}^{+}$. Then we have

$$
\frac{p}{q}-\sum_{j=1}^{R} A_{j} \prod_{n=1}^{N-1}\left(1+\frac{b_{j, n}}{a_{j, n}}\right)=\sum_{j=1}^{R} A_{j} \prod_{n=1}^{\infty}\left(1+\frac{b_{j, n}}{a_{j, n}}\right)-\sum_{j=1}^{R} A_{j} \prod_{n=1}^{N-1}\left(1+\frac{b_{j, n}}{a_{j, n}}\right) .
$$

So the number

$$
\begin{gathered}
\alpha(N)=q\left(\prod_{j=1}^{R} \prod_{n=1}^{N-1} a_{j, n}\right)\left|\frac{p}{q}-\sum_{j=1}^{R} A_{j} \prod_{n=1}^{N-1}\left(1+\frac{b_{j, n}}{a_{j, n}}\right)\right|= \\
q\left(\prod_{j=1}^{R} \prod_{n=1}^{N-1} a_{j, n}\right)\left|\sum_{j=1}^{R} A_{j}\left(\prod_{n=1}^{N-1}\left(1+\frac{b_{j, n}}{a_{j, n}}\right)\right)\left(\prod_{n=N}^{\infty}\left(1+\frac{b_{j, n}}{a_{j, n}}\right)-1\right)\right|
\end{gathered}
$$


is an integer. To prove our theorem it is enough to prove that there exists $N_{0} \in \mathbb{Z}^{+} \backslash\{1\}$ such that $0<\alpha\left(N_{0}\right)<1$. Let us assume that $N$ is sufficiently large. Set

$$
D=\max _{j \in\{1,2, \ldots, K\}}\left|A_{j}\right| \prod_{n=1}^{\infty}\left(1+\frac{b_{j, n}}{a_{j, n}}\right) .
$$

From this, the facts that $N$ is sufficiently large and $\prod_{n=1}^{\infty}\left(1+\frac{b_{j, n}}{a_{j, n}}\right)<\infty$ for all $j=1, \ldots, K$ we obtain that $D$ is positive real number and $\prod_{n=N}^{\infty}\left(1+\frac{b_{j, n}}{a_{j, n}}\right)<$ $2 \sum_{n=N}^{\infty} \frac{b_{j, n}}{a_{j, n}}$ holds for every $j=1, \ldots, K$.

1. Now we prove that $\alpha(N)>0$ for all large $N$. From (7) and the fact that $\prod_{n=N}^{\infty}\left(1+\frac{b_{j, n}}{a_{j, n}}\right)<2 \sum_{n=N}^{\infty} \frac{b_{j, n}}{a_{j, n}}$ holds for every $j=1, \ldots, K$ we obtain that

$$
\begin{gathered}
\alpha(N)\left(q \prod_{j=1}^{R} \prod_{n=1}^{N-1} a_{j, n}\right)^{-1}=\left|\sum_{j=1}^{R} A_{j}\left(\prod_{n=1}^{N-1}\left(1+\frac{b_{j, n}}{a_{j, n}}\right)\right)\left(\prod_{n=N}^{\infty}\left(1+\frac{b_{j, n}}{a_{j, n}}\right)-1\right)\right| \geq \\
\left|A_{R}\right|\left(\prod_{n=1}^{N-1}\left(1+\frac{b_{R, n}}{a_{R, n}}\right)\right)\left(\prod_{n=N}^{\infty}\left(1+\frac{b_{R, n}}{a_{R, n}}\right)-1\right)- \\
\sum_{j=1}^{R-1}\left|A_{j}\right|\left(\prod_{n=1}^{N-1}\left(1+\frac{b_{j, n}}{a_{j, n}}\right)\right)\left(\prod_{n=N}^{\infty}\left(1+\frac{b_{j, n}}{a_{j, n}}\right)-1\right)> \\
\left(\prod_{n=N}^{\infty}\left(1+\frac{b_{R, n}}{a_{R, n}}\right)-1\right)-D \sum_{j=1}^{R-1}\left(\prod_{n=N}^{\infty}\left(1+\frac{b_{j, n}}{a_{j, n}}\right)-1\right)> \\
\frac{1}{2} \sum_{n=N}^{\infty} \frac{b_{R, n}}{a_{R, n}}-2 D \sum_{j=1}^{R-1} \sum_{n=N}^{\infty} \frac{b_{j, n}}{a_{j, n}}= \\
\sum_{n=N}^{\infty} \frac{b_{R, n}}{a_{R, n}}\left(\frac{1}{2}-2 D \sum_{j=1}^{R-1} \frac{b_{j, n} a_{R, n}}{a_{j, n} b_{R, n}}\right) .
\end{gathered}
$$

This and (2) imply that $\alpha(N)>0$ for all large $N$.

2. Now we prove that $\alpha(N)<1$ for infinitely many large $N$. To prove this we will estimate the product $\prod_{j=1}^{R} \prod_{n=1}^{N-1} a_{j, n}$. From the definition of $F$ we obtain that

$$
a_{1, n}<(2 F)^{(K+1)^{n}}
$$


for all sufficiently large $n$. Inequality (5) implies that

$$
\prod_{j=1}^{R} \prod_{n=1}^{N-1} a_{j, n} \leq D_{1} \prod_{j=1}^{R} \prod_{n=1}^{N-1} a_{1, n} a_{1, n}^{\frac{1}{\log _{2}^{1+\varepsilon} \log _{2} a_{1, n}}}
$$

where $D_{1}$ is a suitable constant which does not depend on $N$. Recall that the notation $\log _{2}^{a} \log _{2} x$ means $\left(\log _{2}\left(\log _{2} x\right)\right)^{a}$. This, (8) and the fact that the function $x^{\left(\log _{2} \log _{2} x\right)^{-(1+\varepsilon)}}$ is increasing for all sufficiently large $x$ yield

$$
\begin{gathered}
\prod_{j=1}^{R} \prod_{n=1}^{N-1} a_{j, n} \leq D_{1} \prod_{j=1}^{R} \prod_{n=1}^{N-1} a_{1, n} a_{1, n}^{\frac{1}{\log _{2}^{1+\varepsilon} \log _{2} a_{1, n}}} \leq \\
D_{2}\left(\prod_{n=1}^{N-1} a_{1, n}\right)^{K}\left(\prod_{n=1}^{N-1} 2^{\frac{D_{3}}{n^{1+\varepsilon}}(K+1)^{n}}\right)= \\
D_{2}\left(\prod_{n=1}^{N-1} a_{1, n}\right)^{K}\left(2^{D_{3} \sum_{n=1}^{N-1} \frac{1}{n^{1+\varepsilon}}(K+1)^{n}}\right) \leq 2^{N^{-\left(1+\frac{\varepsilon}{2}\right)}}(K+1)^{N}\left(\prod_{n=1}^{N-1} a_{1, n}\right)^{K}
\end{gathered}
$$

where $D_{2}$, and $D_{3}$ are positive real constants which do not depend on $N$. From (3) and (5) we obtain that

$$
\begin{aligned}
& \left|\sum_{j=1}^{R} A_{j}\left(\prod_{n=1}^{N-1}\left(1+\frac{b_{j, n}}{a_{j, n}}\right)\right)\left(\prod_{n=N}^{\infty}\left(1+\frac{b_{j, n}}{a_{j, n}}\right)-1\right)\right| \leq \\
& D \sum_{j=1}^{R}\left(\prod_{n=N}^{\infty}\left(1+\frac{b_{j, n}}{a_{j, n}}\right)-1\right) \leq 2 D \sum_{j=1}^{R} \sum_{n=N}^{\infty} \frac{b_{j, n}}{a_{j, n}} \leq 2 D K \sum_{n=N}^{\infty} a_{1, n}^{\frac{2}{\log _{2}^{1+\varepsilon} \log _{2} a_{1, n}}-1} \leq \\
& \sum_{n=N}^{\infty} a_{1, n}^{\frac{1}{\log _{2} \frac{\varepsilon}{2}} \log _{2} a_{1, n}}-1
\end{aligned}
$$

From this, (7) and (9) we obtain for all sufficiently large $N$

$$
\begin{gathered}
\alpha(N)=q\left(\prod_{j=1}^{R} \prod_{n=1}^{N-1} a_{j, n}\right)\left|\sum_{j=1}^{R} A_{j}\left(\prod_{n=1}^{N-1}\left(1+\frac{b_{j, n}}{a_{j, n}}\right)\right)\left(\prod_{n=N}^{\infty}\left(1+\frac{b_{j, n}}{a_{j, n}}\right)-1\right)\right| \leq \\
q 2^{N^{-\left(1+\frac{\varepsilon}{2}\right)}(K+1)^{N}}\left(\prod_{n=1}^{N-1} a_{1, n}\right)^{K} \sum_{n=N}^{\infty} a_{1, n}^{\log _{2}^{-\left(1+\frac{\varepsilon}{2}\right)} \log _{2} a_{1, n}-1} .
\end{gathered}
$$

Let $T_{n}=a_{1, n}^{\frac{1}{(K+1)^{n}}}$. Now the proof falls into two cases. 
2a. First assume that for every sufficiently large $n$

$$
a_{1, n} \geq 2^{n}
$$

Then (11) and the fact that the function $x^{\log _{2}^{-\left(1+\frac{\varepsilon}{2}\right)} \log _{2} x-1}$ is decreasing for sufficiently large $x$ imply

$$
\begin{gathered}
\sum_{n=N}^{\infty} a_{1, n}^{\log _{2}^{-\left(1+\frac{\varepsilon}{2}\right)} \log _{2} a_{1, n}-1}= \\
\sum_{N \leq n \leq \log _{2} a_{1, N}} a_{1, n}^{\log _{2}^{-\left(1+\frac{\varepsilon}{2}\right)} \log _{2} a_{1, n}-1}+\sum_{n>\log _{2} a_{1, N}} a_{1, n}^{\log _{2}^{-\left(1+\frac{\varepsilon}{2}\right)} \log _{2} a_{1, n}-1} \leq \\
a_{1, N}^{\log _{2}^{-\left(1+\frac{\varepsilon}{2}\right)} \log _{2} a_{1, N}-1} \log _{2} a_{1, N}+\sum_{n>\log _{2} a_{1, N}} a_{1, n}^{\log _{2}^{-\left(1+\frac{\varepsilon}{2}\right)} \log _{2} a_{1, n}-1} \leq \\
a_{1, N}^{\log _{2}^{-\left(1+\frac{\varepsilon}{2}\right)} \log _{2} a_{1, N}-1} \log _{2} a_{1, N}+\sum_{n>\log _{2} a_{1, N}} 2^{n\left(\log _{2}^{-\left(1+\frac{\varepsilon}{2}\right)} n-1\right)} \leq \\
a_{1, N}^{\log _{2}^{-\left(1+\frac{\varepsilon}{2}\right)} \log _{2} a_{1, N}-1} \log _{2} a_{1, N}+\sum_{n>\log _{2} a_{1, N}} 2^{n\left(\log _{2}^{-\left(1+\frac{\varepsilon}{2}\right)} \log _{2} a_{1, N}-1\right)} \leq \\
a_{1, N}^{\log _{2}^{-\left(1+\frac{\varepsilon}{2}\right)} \log _{2} a_{1, N}-1} \log _{2}^{-\left(1+\frac{\varepsilon}{4}\right)} a_{\log _{2} a_{1, N}-1} .
\end{gathered}
$$

for sufficiently large $N$.

For a sufficiently small positive real number $\delta$, from (5) it follows that there exists a positive integer $t_{0}$ which is sufficiently large such that for every $n \geq t_{0}$ we have $\max (1, E-\delta)<T_{n}<F+\delta$. This implies that for every $n \geq t_{0}$

$$
\max (1,(E-\delta))^{(K+1)^{n}}<a_{1, n}<(F+\delta)^{(K+1)^{n}} .
$$

Let $t_{1}$ be the least positive integer greater than $(K+1)^{t_{0}+1}$ such that the inequality $\max (1, E-\delta)<T_{t_{1}}<E+\delta$ holds. Then

$$
\max (1,(E-\delta))^{(K+1)^{t_{1}}}<a_{1, t_{1}}<(E+\delta)^{(K+1)^{t_{1}}} .
$$

Let $t_{2}$ be the least positive integer greater than $t_{1}$ such that

$$
F-\delta<T_{t_{2}}<F+\delta
$$


and let $t_{3}$ be the least positive integer greater than $t_{1}$ such that $t_{1}<t_{3} \leq t_{2}$ and

$$
T_{t_{3}}>\left(1+\frac{1}{t_{3}^{1+\frac{\varepsilon}{6}}}\right) \max _{t_{1} \leq j<t_{3}}\left(T_{j}, F-2 \delta\right) .
$$

Such a number $t_{3}$ must exist since otherwise using (15) we obtain

$$
\begin{gathered}
F-\delta<T_{t_{2}} \leq\left(1+\frac{1}{t_{2}^{1+\frac{\varepsilon}{6}}}\right) \max _{t_{1} \leq j<t_{2}}\left(T_{j}, F-2 \delta\right) \leq \\
\left(1+\frac{1}{t_{2}^{1+\frac{\varepsilon}{6}}}\right)\left(1+\frac{1}{\left(t_{2}-1\right)^{1+\frac{\varepsilon}{6}}}\right) \max _{t_{1} \leq j<t_{2}-1}\left(T_{j}, F-2 \delta\right)<\ldots \\
<\prod_{j=t_{1}}^{t_{2}}\left(1+\frac{1}{j^{1+\frac{\varepsilon}{6}}}\right)(F-2 \delta),
\end{gathered}
$$

a contradiction for a sufficiently large $t_{0}$. From (8),(13), (14) and (16) we obtain

$$
\begin{aligned}
& a_{1, t_{3}}=T_{t_{3}}^{(K+1)^{t_{3}}}>\left(1+\frac{1}{t_{3}^{1+\frac{\varepsilon}{6}}}\right)^{(K+1)^{t_{3}}}\left(\max _{t_{1} \leq j<t_{3}}\left(T_{j}, F-2 \delta\right)\right)^{(K+1)^{t_{3}}} \geq \\
& \left(1+\frac{1}{t_{3}^{1+\frac{\varepsilon}{6}}}\right)^{(K+1)^{t_{3}}} \max _{t_{1} \leq j<t_{3}}\left(T_{j}, F-2 \delta\right)^{K\left((K+1)^{t_{3}-1}+(K+1)^{t_{3}-2}+\cdots+1\right)} \geq \\
& \left(1+\frac{1}{t_{3}^{1+\frac{\varepsilon}{6}}}\right)^{(K+1)^{t_{3}}}\left(\prod_{j=t_{1}+1}^{t_{3}-1} a_{1, j}\right)^{K}(F-2 \delta)^{K\left((K+1)^{t_{1}}+(K+1)^{t_{1}-1}+\cdots+1\right)} \geq \\
& \left(1+\frac{1}{t_{3}^{1+\frac{\varepsilon}{6}}}\right)^{(K+1)^{t_{3}}}\left(\prod_{j=1}^{t_{3}-1} a_{1, j}\right)^{K} \prod_{j=t_{0}}^{t_{1}}\left(\frac{(F-2 \delta)^{(K+1)^{j}}}{a_{1, j}}\right)^{K} \frac{1}{\left(\prod_{j=1}^{t_{0}-1} a_{1, j}\right)^{K}} \geq \\
& \left(1+\frac{1}{t_{3}^{1+\frac{\varepsilon}{6}}}\right)^{(K+1)^{t_{3}}}\left(\prod_{j=1}^{t_{3}-1} a_{1, j}\right)^{K}\left(\frac{F-2 \delta}{E+\delta}\right)^{K(K+1)^{t_{1}}} \times \\
& \prod_{j=t_{0}}^{t_{1}-1}\left(\left(\frac{F-2 \delta}{F+\delta}\right)^{(K+1)^{j}}\right)^{K} \frac{D_{4}}{\prod_{j=1}^{t_{0}-1}(2 F)^{K(K+1)^{j}}} \geq \\
& \left(1+\frac{1}{t_{3}^{1+\frac{\varepsilon}{6}}}\right)^{(K+1)^{t_{3}}}\left(\prod_{j=1}^{t_{3}-1} a_{1, j}\right)^{K}\left(\prod_{j=t_{0}}^{t_{1}-1}\left(\frac{(F-2 \delta)^{2}}{(E+\delta)(F+\delta)}\right)^{(K+1)^{j}}\right)^{K} \times \\
& (3 F)^{-(K+1)^{t_{0}+1}} \geq
\end{aligned}
$$




$$
\left(1+\frac{1}{t_{3}^{1+\frac{\varepsilon}{6}}}\right)^{(K+1)^{t_{3}}}\left(\prod_{j=1}^{t_{3}-1} a_{1, j}\right)^{K}(3 F)^{-t_{3}}
$$

where $D_{4}$ is a positive real constant which does not depend on $t_{0}$. Now from (8), (10), (12), and (17) we obtain

$$
\begin{aligned}
& \alpha\left(t_{3}\right) \leq q 2^{t_{3}^{-\left(1+\frac{\varepsilon}{2}\right)}}(K+1)^{t_{3}}\left(\prod_{n=1}^{t_{3}-1} a_{1, n}\right)^{K} \sum_{n=t_{3}}^{\infty} a_{1, n}^{\log _{2}^{-\left(1+\frac{\varepsilon}{2}\right)} \log _{2} a_{1, n}-1} \leq \\
& q 2^{t_{3}^{-\left(1+\frac{\varepsilon}{2}\right)}(K+1)^{t_{3}}}\left(\prod_{n=1}^{t_{3}-1} a_{1, n}\right)^{K} a_{1, t_{3}}^{\log _{2}^{-\left(1+\frac{\varepsilon}{4}\right)} \log _{2} a_{1, t_{3}-1} \leq} \\
& q 2^{t_{3}^{-\left(1+\frac{\varepsilon}{2}\right)}(K+1)^{t_{3}}}\left(\prod_{n=1}^{t_{3}-1} a_{1, n}\right)^{K} \frac{a_{1, t_{3}}^{\log _{2}^{-\left(1+\frac{\varepsilon}{4}\right)}} \log _{2} a_{1, t_{3}}}{\left(1+\frac{1}{t_{3}^{1+\frac{\varepsilon}{6}}}\right)^{(K+1)^{t_{3}}}\left(\prod_{j=1}^{t_{3}-1} a_{1, j}\right)^{K}(3 F)^{-t_{3}}} \leq \\
& 2^{t_{3}^{-\left(1+\frac{\varepsilon}{2}\right)}(K+1)^{t_{3}}} \frac{2^{t_{3}^{-\left(1+\frac{\varepsilon}{5}\right)}(K+1)^{t_{3}}}}{\left(1+\frac{1}{t_{3}^{1+\frac{\varepsilon}{6}}}\right)^{(K+1)^{t_{3}}}(3 F)^{-t_{3}}}= \\
& 2^{t_{3}^{-\left(1+\frac{\varepsilon}{2}\right)}(K+1)^{t_{3}}+t_{3}^{-\left(1+\frac{\varepsilon}{5}\right)}(K+1)^{t_{3}}-\log _{2}\left(1+\frac{1}{t_{3}+\frac{\varepsilon}{6}}\right)(K+1)^{t_{3}}+t_{3} \log _{2}(3 F)}<1
\end{aligned}
$$

for a sufficiently large number $t_{3}$.

2 b. Now assume that there exist infinitely many $n$ such that

$$
a_{1, n}<2^{n} \text {. }
$$

Then (4) and the fact that the function $x^{\log _{2}^{-\left(1+\frac{\varepsilon}{2}\right)} \log _{2} x-1}$ is decreasing for sufficiently large $x$ imply

$$
\begin{gathered}
\sum_{n=N}^{\infty} a_{1, n}^{\log _{2}^{-\left(1+\frac{\varepsilon}{2}\right)} \log _{2} a_{1, n}-1} \leq \\
\sum_{N \leq n \leq \sqrt[5]{a_{1, N}}} a_{1, n}^{\log _{2}^{-\left(1+\frac{\varepsilon}{2}\right)} \log _{2} a_{1, n}-1}+\sum_{n>\sqrt[5]{a_{1, N}}} a_{1, n}^{\log _{2}^{-\left(1+\frac{\varepsilon}{2}\right)} \log _{2} a_{1, n}-1} \leq \\
a_{1, N}^{\log _{2}^{-\left(1+\frac{\varepsilon}{2}\right)} \log _{2} a_{1, N}-1} \sqrt[5]{a_{1, N}}+\sum_{n>\sqrt[5]{a_{1, N}}} a_{1, n}^{\log _{2}^{-\left(1+\frac{\varepsilon}{2}\right)} \log _{2} a_{1, n}-1} \leq
\end{gathered}
$$




$$
\begin{aligned}
& a_{1, N}^{\log _{2}^{-\left(1+\frac{\varepsilon}{2}\right)} \log _{2} a_{1, N}-1} \sqrt[5]{a_{1, N}}+\sum_{n>\sqrt[5]{a_{1, N}}} n^{(1+\varepsilon)\left(\log _{2}^{-\left(1+\frac{\varepsilon}{2}\right)} \log _{2} n^{1+\varepsilon}-1\right)} \leq \\
& a_{1, N}^{\log _{2}^{-\left(1+\frac{\varepsilon}{2}\right)} \log _{2} a_{1, N}-1} \sqrt[5]{a_{1, N}}+a_{1, N}^{-\frac{\varepsilon}{10}} \leq \\
& a_{1, N}^{-A},
\end{aligned}
$$

for sufficiently large $N$, where $A=\frac{1}{20} \min (1, \varepsilon)$. Now, set $B=\frac{1}{2}(1+F)=$ $\frac{1}{2}(E+F)$. From this and (1) we obtain that there is a sufficiently large $k$ such that

$$
a_{1, k}>B^{(K+1)^{k}} .
$$

Let $k_{0}$ be the greatest positive integer less than $k$ such that (18) holds. Let $k_{1}$ be the least positive integer such that

$$
T_{k_{1}}>\left(1+\frac{1}{k_{1}^{1+\frac{\varepsilon}{6}}}\right) \max _{k_{0} \leq j<k_{1}} T_{j},
$$

and $k_{0}<k_{1} \leq k$. As in the previous case such a $k_{1}$ must exist since otherwise

$$
\begin{aligned}
& 1<B \leq T_{k} \leq\left(1+\frac{1}{k^{1+\frac{\varepsilon}{6}}}\right) \max _{k_{0} \leq j<k} T_{j}< \\
&\left(1+\frac{1}{k^{1+\frac{\varepsilon}{6}}}\right)\left(1+\frac{1}{(k-1)^{1+\frac{\varepsilon}{6}}}\right) \max _{k_{0} \leq j<k-1} T_{j}<\cdots< \\
&<\prod_{j=k_{0}+1}^{k}\left(1+\frac{1}{j^{1+\frac{\varepsilon}{6}}}\right) T_{k_{0}},
\end{aligned}
$$

a contradiction for a sufficiently large number $k_{0}$. From (21) and the fact that the sequence $\left\{a_{1, n}\right\}_{n=1}^{\infty}$ is non-decreasing we obtain

$$
\begin{gathered}
a_{1, k_{1}}=T_{k_{1}}^{(K+1)^{k_{1}}}>\left(1+\frac{1}{k_{1}^{1+\frac{\varepsilon}{6}}}\right)^{(K+1)^{k_{1}}}\left(\max _{k_{0} \leq j<k_{1}} T_{j}\right)^{(K+1)^{k_{1}}} \geq \\
\left(1+\frac{1}{k_{1}^{1+\frac{\varepsilon}{6}}}\right)^{(K+1)^{k_{1}}}\left(\max _{k_{0} \leq j<k_{1}} T_{j}\right)^{K\left((K+1)^{k_{1}-1}+(K+1)^{k_{1}-2}+\cdots+1\right)} \geq \\
\left(1+\frac{1}{k_{1}^{1+\frac{\varepsilon}{6}}}\right)^{(K+1)^{k_{1}}}\left(\prod_{j=1}^{k_{1}-1} a_{1, j}\right)^{K}\left(\prod_{j=1}^{k_{0}} a_{1, j}\right)^{-K} \geq \\
\left(1+\frac{1}{k_{1}^{1+\frac{\varepsilon}{6}}}\right)^{(K+1)^{k_{1}}}\left(\prod_{j=1}^{k_{1}-1} a_{1, j}\right)^{K} 2^{-K k_{0}^{2}} .
\end{gathered}
$$


The definition of $k_{1}$ implies that for every $N\left(k_{0}<N<k_{1}\right)$

$$
T_{N} \leq\left(1+\frac{1}{N^{1+\frac{\varepsilon}{6}}}\right) \max _{k_{0} \leq j<N} T_{j} .
$$

Thus

$$
T_{N} \leq\left(\prod_{j=k_{0}+1}^{N}\left(1+\frac{1}{j^{1+\frac{\varepsilon}{6}}}\right)\right) T_{k_{0}}<\left(\prod_{j=k_{0}}^{\infty}\left(1+\frac{1}{j^{1+\frac{\varepsilon}{6}}}\right)\right) T_{k_{0}}=C=C\left(k_{0}\right),
$$

where $C$ is a constant which depends on $k_{0}$ and $C$ tends to 1 as $k_{0}$ tends to infinity. From (23) we obtain that for every $N=k_{0}, \ldots, k_{1}-1$

$$
a_{1, N} \leq C^{(K+1)^{N}} .
$$

This yields

$$
\left(\prod_{j=1}^{k_{1}-1} a_{1, j}\right)^{K}=\left(\prod_{j=1}^{k_{0}} a_{1, j}\right)^{K}\left(\prod_{j=k_{0}}^{k_{1}-1} a_{1, j}\right)^{K} \leq 2^{K k_{0}^{2}} C^{(K+1)^{k_{1}}} .
$$

Inequalities (12) and (19), and the definition of $k_{1}$ and $k$ imply

$$
\begin{gathered}
\sum_{n=k_{1}}^{\infty} a_{1, n}^{\log _{2}^{-\left(1+\frac{\varepsilon}{2}\right)} \log _{2} a_{1, n}-1}= \\
\sum_{n=k_{1}}^{k-1} a_{1, n}^{\log _{2}^{-\left(1+\frac{\varepsilon}{2}\right)} \log _{2} a_{1, n}-1}+\sum_{n=k}^{\infty} a_{1, n}^{\log _{2}^{-\left(1+\frac{\varepsilon}{2}\right)} \log _{2} a_{1, n}-1} \leq \\
a_{1, k_{1}}^{\log _{2}^{-\left(1+\frac{\varepsilon}{4}\right)} \log _{2} a_{1, k_{1}}-1}+a_{1, k}^{-A} .
\end{gathered}
$$

Now from (7), (8), (10), (20), (22), (24), and (25) we obtain

$$
\begin{array}{r}
\alpha\left(k_{1}\right) \leq q 2^{k_{1}^{-\left(1+\frac{\varepsilon}{2}\right)}(K+1)^{k_{1}}}\left(\prod_{n=1}^{k_{1}-1} a_{1, n}\right)^{K} \sum_{n=k_{1}}^{\infty} a_{1, n}^{\log _{2}^{-\left(1+\frac{\varepsilon}{2}\right)} \log _{2} a_{1, n}-1} \leq \\
2^{k_{1}^{-\left(1+\frac{\varepsilon}{3}\right)}(K+1)^{k_{1}}}\left(\prod_{n=1}^{k_{1}-1} a_{1, n}\right)^{K}\left(a_{1, k_{1}}^{\log _{2}^{-\left(1+\frac{\varepsilon}{4}\right)} \log _{2} a_{1, k_{1}}-1}+a_{1, k}^{-A}\right)= \\
\frac{\left(\prod_{n=1}^{k_{1}-1} a_{1, n}\right)^{K} 2^{k_{1}^{-\left(1+\frac{\varepsilon}{3}\right)}(K+1)^{k_{1}}} a_{1, k_{1}}^{\log _{2}^{-\left(1+\frac{\varepsilon}{4}\right)} \log _{2} a_{1, k_{1}}}+}{a_{1, k_{1}}} \leq \\
\frac{\left(\prod_{n=1}^{k_{1}-1} a_{1, n}\right)^{K} 2^{k_{1}^{-\left(1+\frac{\varepsilon}{3}\right)}(K+1)^{k_{1}}}}{a_{1, k}^{A}} \leq
\end{array}
$$




$$
\begin{aligned}
& \frac{\left(\prod_{n=1}^{k_{1}-1} a_{1, n}\right)^{K} 2^{k_{1}^{-\left(1+\frac{\varepsilon}{3}\right)}}(K+1)^{k_{1}} a_{1, k_{1}}^{\log _{2}^{-\left(1+\frac{\varepsilon}{4}\right)} \log _{2} a_{1, k_{1}}}}{\left(1+\frac{1}{k_{1}^{1+\frac{\varepsilon}{6}}}\right)^{(K+1)^{k_{1}}}\left(\prod_{j=1}^{k_{1}-1} a_{1, j}\right)^{K} 2^{-K k_{0}^{2}}}+ \\
& \frac{2^{K k_{0}^{2}} C^{(K+1)^{k_{1}}} 2^{k_{1}^{-\left(1+\frac{\varepsilon}{3}\right)}(K+1)^{k_{1}}}}{B^{A(K+1)^{k}}} \leq \\
& \frac{2^{k_{1}^{-\left(1+\frac{\varepsilon}{3}\right)}(K+1)^{k_{1}}} 2^{k_{1}^{-\left(1+\frac{\varepsilon}{5}\right)}(K+1)^{k_{1}}}}{\left(1+\frac{1}{k_{1}^{1+\frac{\varepsilon}{6}}}\right)^{(K+1)^{k_{1}}} 2^{-K k_{0}^{2}}}+\frac{2^{K k_{0}^{2}} C^{(K+1)^{k_{1}}} 2^{k_{1}^{-\left(1+\frac{\varepsilon}{3}\right)}(K+1)^{k_{1}}}}{B^{A(K+1)^{k}}}<1,
\end{aligned}
$$

a contradiction for a sufficiently large $k_{0}$.

We would like to thank Professor James E. Carter of the College of Charleston for his help with the presentation of this paper. The authors also thank referee for the valuable suggestions.

\section{References}

[1] Erdős P, Some Problems and Results on the Irrationality of the Sum of Infinite Series, J. Math. Sci. 10 (1975), 1-7.

[2] Erdős P, Erdős problem no. 6, 1995 Prague Midsummer Combinatorial Workshop, KAM Series (95-309), M. Klazar (ed.), (1995), page 5.

[3] Hančl J., A criterion for linear independence of series, Rocky Mountain J. Math. 34, no. 1, (2004), 173-186.

[4] Hančl J., Kolouch O, Erdös' method for determining the irrationality of products, Bull. Aust. Math. Soc. 84, no. 3, (2011), 414-424.

[5] Hančl J., Sobková S, Special linearly unrelated sequences, J. Math. Kyoto Univ., vol. 46, no. 1, (2006), 31-45.

[6] Nishioka K, Mahler functions and transcendence, Lecture notes in mathematics 1631, Springer, (1996).

[7] Šustek J, New bounds for irrationality measures of some fast converging series, Mathematics for Applications, vol. 3, no. 2, (2014), 167-175.

Jaroslav HANČL,

Department of Mathematics and Centre of Excellence IT4Innovation, division

of UO, Institute for Research and Applications of Fuzzy Modeling

University of Ostrava

30. dubna 22, 70103 Ostrava 1, Czech Republic.

Email: hancl@osu.cz 
Ondřej KOLOUCH

Department of Mathematics

Faculty of Science

University of Ostrava

30. Dubna 22, 70103 Ostrava 1,Czech republic.

Email: ondrej.kolouch@osu.cz

Lukáš NOVOTNÝ

Department of Mathematics

Faculty of Science

University of Ostrava

30. Dubna 22, 70103 Ostrava 1,Czech republic.

Email: lukas.novotny@osu.cz 